\title{
Discinergia Ventricular Esquerda Reversível Identificada por Potenciação Pós-Extrassistólica em Miocardiopatia Chagásica Crônica Não é Causada por Hibernação Miocárdica
}

\author{
Andrea Miziara', José Antonio Marin-Neto', Júlio Flávio Meirelles Marchini', Geraldo L. Figueiredo', \\ Antonio Oswaldo Pintya ${ }^{1}$, Marcus V. Simões ${ }^{1}$, Cleyde M. Antloga ${ }^{1}$
}

\section{RESUMO}

Introdução: A alteração regional da mobilidade segmentar do ventrículo esquerdo é marcador precoce de miocardiopatia chagásica crônica. Demonstramos recentemente que a discinergia em algumas regiões ventriculares pode ser revertida pela potenciação pós-extrassistólica. Apesar de angiografia coronária normal, pacientes com miocardiopatia chagásica apresentam falhas de perfusão, corroborando a hipótese de que a hibernação miocárdica pode ser responsável pelas anormalidades de mobilidade segmentar revertidas durante a potenciação pós-extrassistólica. Método: Vinte e dois pacientes consecutivos portadores de miocardiopatia chagásica foram submetidos a angiografia coronária, ventriculografia e cintilografia miocárdica com tálio-201 com o protocolo estresse-redistribuição-reinjeção para avaliação da perfusão dos segmentos do ventrículo esquerdo demonstrando potenciação pós-extrassistólica. Resultados: Não foram observadas obstruções coronárias significativas nos pacientes avaliados. Potenciação pós-extrassistólica ocorreu em pelo menos um segmento ventricular esquerdo em 14 dos 22 pacientes, sendo observada em segmentos ventriculares com diminuição de mobilidade de moderada a intensa, mas não em segmentos com discinesia. Esses pacientes apresentaram melhora da média da pontuação de mobilidade segmentar de $1,49 \pm 0,73$ para 1,36 $\pm 0,76$ do batimento basal ao batimento pós-extrassistólico $(P<0,05)$. Nessas áreas, a perfusão miocárdica era normal ou apenas levemente prejudicada e sem demonstração de isquemia com exercício ou estresse farmacológico. Conclusão: Estes resultados não corroboram a hipótese de miocárdio

\section{ABSTRACT}

\section{Reversible Dysynergy Identified by \\ Post-Extrasystolic Potentiation in Chronic Chagas \\ Cardiomyopathy Is Not Caused by Myocardial Hibernation}

Background: Regional left ventricular segmental wall motion impairment is an early marker of chronic Chagas cardiomyopathy. We have recently shown that dysynergy may be reversed in some ventricular regions by post-extrasystolic potentiation. Despite normal epicardial coronary arteries, patients with Chagas cardiomyopathy have perfusion defects, raising the possibility that myocardial hibernation could be responsible for the wall motion abnormalities reversed during post-extrasystolic potentiation. Methods: Twentytwo consecutive patients with chronic Chagas cardiomyopathy underwent coronary angiography, left ventricular contrast angiography and stress-redistribution-reinjection thallium-201 myocardial scintigraphy for the assessment of the perfusion status in left ventricular segments showing the presence of post-extrasystolic potentiation. Results: No significant coronary artery stenosis was seen in any of the patients. Post-extrasystolic potentiation was seen in at least one left ventricular segment in 14 of the 22 patients. It occurred in ventricular areas with baseline moderate to intense wall motion disorders, but not with dyskinesis. These patients showed an improvement in the average myocardial wall motion scores of $1.49 \pm 0.73$ to $1.36 \pm 0.76$ from the baseline to the post-extrasystolic beat $(P<0.05)$. In these areas, myocardial perfusion was normal or only mildly impaired, and ischemia was not induced by exercise or

N. da E. - A grafia discinergia (e suas variações) foi mantida conforme o original.

Projeto parcialmente financiado pelo CNPq (Processo № 520478/95-9) e pela FAPESP (Processo № 1995/6195-8).

1 Hospital das Clínicas da Faculdade de Medicina de Ribeirão Preto (USP) - Ribeirão Preto, SP, Brasil.

Correspondência: José Antonio Marin-Neto. Rua João Gomes Rocha, 880/201 - Jardim Irajá - Ribeirão Preto, SP, Brasil - CEP 14020-550 E-mail: marin neto@yahoo.com

Recebido em: 25/6/2009 • Aceito em: 11/9/2009 
Miziara A, et al. Discinergia Ventricular Esquerda Reversível Identificada por Potenciação Pós-Extrassistólica em Miocardiopatia Chagásica Crônica Não é Causada por Hibernação Miocárdica. Rev Bras Cardiol Invas. 2009;17(3):358-68.

hibernante causado por distúrbio de perfusão microvascular na miocardiopatia chagásica.

DESCRITORES: Doença de Chagas/fisiopatologia. Contração miocárdica/fisiopatologia. Estimulação elétrica/métodos.

A alteração regional da mobilidade segmentar dos ventrículos esquerdo e direito é um marcador de miocardiopatia chagásica crônica ${ }^{1}$. Predominantes nas regiões apical e póstero-lateral do ventrículo esquerdo, esses distúrbios de mobilidade são achados precoces na história natural da doença e estão associados a arritmias, podendo levar a morte súbita². Entretanto, ao contrário do que acontece com pacientes com doença coronária, as alterações da mobilidade ventricular em pacientes chagásicos não são causadas por obstruções coronárias detectáveis por angiografia ${ }^{3,4}$. Apesar da ausência de lesões coronárias epicárdicas, observaram-se falhas de perfusão regional nas mesmas áreas que tinham alterações de mobilidade ventricular em pacientes chagásicos avaliados por cintilografia do miocárdio.

Utilizando ventriculografia de contraste, relatamos recentemente que é possível demonstrar a presença de reserva contrátil por potenciação pós-extrassistólica em áreas com discinergia ventricular esquerda em pacientes chagásicos ${ }^{5}$. Com base nesses achados, seria razoável dizer que pode ocorrer isquemia microvascular crônica nessas áreas com discinergia, levando a hibernação do miocárdio ${ }^{6,7}$, em pacientes com miocardiopatia chagásica. O presente estudo tem como objetivo identificar os padrões de perfusão miocárdica regional em repouso e durante esforço em pacientes chagásicos manifestando potenciação pós-extrassistólica em áreas com discinergia ventricular esquerda.

\section{MÉTODO}

Este estudo prospectivo incluiu 22 pacientes com miocardiopatia chagásica crônica, encaminhados para cateterização cardíaca e angiocoronariografia para esclarecer os mecanismos de arritmias ventriculares. Todos os pacientes fizeram ventriculografia por contraste para análise da mobilidade segmentar do ventrículo esquerdo de potenciação pós-extrassistólica e, em sessão separada, dentro de no máximo uma semana, cintilografia de perfusão miocárdica para correlação topográfica com os achados de mobilidade segmentar. Todos os pacientes assinaram o termo de consentimento informado e o protocolo do estudo foi aprovado pelo comitê de ética institucional previamente ao início do estudo.

A média de idade do grupo foi de 50,2 \pm 12 anos, sendo 13 pacientes do sexo masculino. Todos tinham pelo menos dois testes sorológicos positivos para doença pharmacological stress. Conclusion: The present results do not support the hypothesis of hibernating myocardium caused by microvascular perfusion disorders in chronic Chagas cardiomyopathy.

DESCRIPTORS: Chagas disease/physiopathology. Myocardial contraction/physiology. Electric stimulation/methods.

de Chagas. Dentre os 22 pacientes, 14 tinham ritmo sinusal com vários tipos de bloqueio de ramo e 8 eram portadores de marca-passo. Todos os pacientes tinham no mínimo uma região do ventrículo esquerdo com alteração da mobilidade segmentar em ecocardiograma bidimensional realizado anteriormente.

\section{Avaliação da potenciação pós-extrassistólica por ventriculografia de contraste}

Após angiocoronariografia pelas técnicas de Sones ou Judkins, foi realizada ventriculografia esquerda com duas injeções sucessivas de 20-35 ml de contraste radiológico no ventrículo esquerdo. A taxa do fluxo de injeção variou de $8 \mathrm{ml} /$ segundo a $15 \mathrm{ml} /$ segundo e a pressão variou de 400 psi a 600 psi, de acordo com o cateter utilizado, respectivamente Sones $8 \mathrm{~F}$ ou pigtail $7 \mathrm{~F}$.

Cada injeção permitiu o registro da ventriculografia esquerda nas posições oblíqua anterior direita a 30 graus e oblíqua anterior esquerda a 45 graus, em cinefilme, com velocidade de 30 quadros/segundo.

A análise da ventriculografia nas posições oblíqua anterior direita e oblíqua anterior esquerda foi realizada por consenso por dois angiógrafos experientes, cegos para os resultados dos exames nucleares. Para isso, adotou-se um sistema semiquantitativo de classificação para cada segmento do ventrículo esquerdo, com a seguinte pontuação: $0=$ mobilidade ventricular normal; 1 = hipocinesia leve; 2 = hipocinesia moderada; 3 = acinesia; e 4 = discinesia.

A análise da mobilidade ventricular foi feita para um modelo de oito segmentos do ventrículo esquerdo, conforme apresentado na Figura 1: anterior basal, ântero-lateral, apical, inferior basal, ínfero-lateral basal, ínfero-lateral médio, inferior e septal ${ }^{8}$. Cada segmento do ventrículo esquerdo recebeu uma pontuação de acordo com seu padrão de mobilidade ventricular durante o batimento sinusal, imediatamente antes e o primeiro batimento sinusal logo após um batimento ventricular ectópico espontâneo depois da injeção de contraste. Calculou-se a pontuação média para os oito segmentos avaliados para cada paciente nos batimentos pré-extrassistólicos e pós-extrassistólicos.

\section{Cintilografia de perfusão miocárdica}

Os pacientes foram orientados a ficar em jejum por aproximadamente seis horas e a não ingerir líqui- 


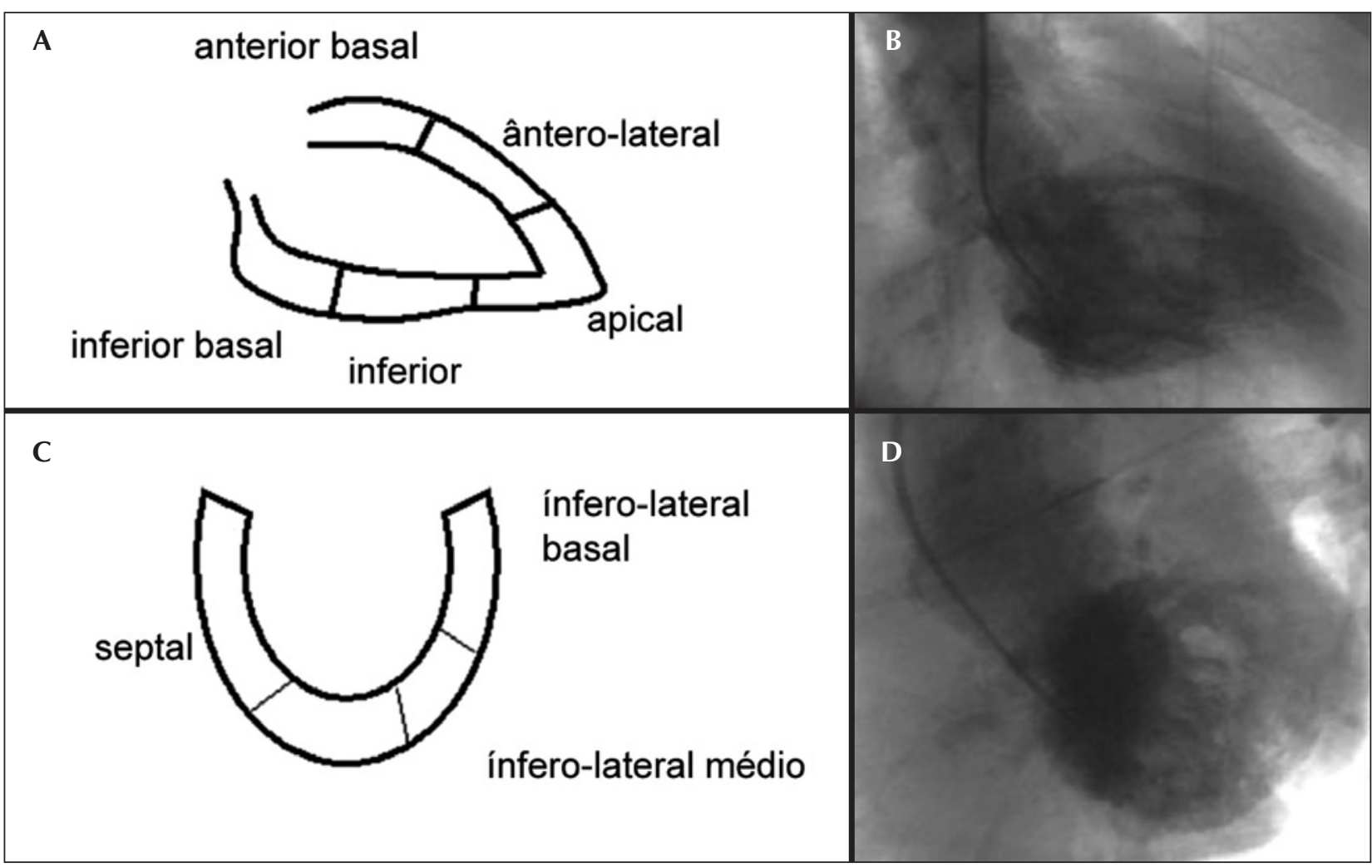

Figura 1 - Esquema de segmentação do ventrículo esquerdo para análise de ventriculografia por contraste. Os painéis A e B referem-se à imagem oblíqua anterior direita e os painéis C e D, à imagem oblíqua anterior esquerda.

dos contendo cafeína ou medicamentos baseados em metilxantinas por 24 horas. Foi realizada cintilografia de perfusão miocárdica SPECT utilizando protocolo de estresse-redistribuição-reinjeção. O estresse foi induzido por esforço físico máximo utilizando o protocolo de Bruce em 16 pacientes, por dipiridamol intravenoso (0,14 mg.kg-1. $\mathrm{min}^{-1}$ por quatro minutos) em 5 pacientes, e por dobutamina intravenosa (doses incrementais de até $40 \mu \mathrm{g} / \mathrm{kg} / \mathrm{min}$ ) no último paciente. Foi injetado tálio-201 (111 MBq) no pico do estresse induzido por esforço ou dois minutos após o final da infusão de dipiridamol, sendo a primeira aquisição realizada dez minutos mais tarde. A segunda aquisição ocorreu três a quatro horas depois, durante a redistribuição do radiomarcador, seguida por reinjeção de $37 \mathrm{MBq}$ de tálio-201 em repouso; e a terceira aquisição foi realizada vinte minutos depois. O eletrocardiograma foi registrado continuamente e a pressão arterial foi medida em intervalos de três minutos durante o teste de esforço e o teste de estresse farmacológico.

Cada aquisição cintilográfica foi realizada utilizando uma câmera de dupla cabeça (DST, Sopha Medical Vision, Twinsburg, Estados Unidos), com detectores retangulares de campo de visão média, equipados com colimadores multiuso de baixa energia. A coleta de dados envolveu 32 projeções - 40 segundos por projeção - em um arco de 180 graus, variando de 45 graus de visão na posição oblíqua anterior direita a 45 graus na posição oblíqua posterior esquerda. A janela de $30 \%$ foi centralizada em $72 \mathrm{keV}$ e a janela de $20 \%$, em $167 \mathrm{keV}$ (fotopicos do tálio-201). As imagens da projeção foram corrigidas para a uniformidade do campo e do centro de rotação, utilizando matrizes de 64 × 64 sem magnificação de aquisição; a reconstrução computadorizada das imagens miocárdicas para cada fase de aquisição foi realizada automaticamente por retroprojeção filtrada com filtro Butterworth (ordem, 5; frequência de corte, 0,25 ciclo/pixel). As imagens de perfusão foram dispostas nas visões do eixo curto e do eixo longo vertical, e também de acordo com a reconstrução do mapa polar, permitindo a análise da perfusão regional de acordo com o modelo padrão de 17 segmentos $^{9}$ apresentado na Figura 2. A inserção do ventrículo direito foi a principal marca anatômica para definir a parede septal.

O padrão de perfusão de cada lâmina de SPECT foi avaliado por dois cardiologistas nucleares experientes, cegos para os resultados da ventriculografia de contraste, utilizando um sistema de classificação semiquantitativo, em que: 0 = perfusão normal; 1 = hipoperfusão leve; 2 = hipoperfusão moderada; 3 = hipoperfusão grave; e 4 = ausência de marcador. As discordâncias iniciais foram solucionadas, posteriormente, por consenso. 
Miziara A, et al. Discinergia Ventricular Esquerda Reversível Identificada por Potenciação Pós-Extrassistólica em Miocardiopatia Chagásica Crônica Não é Causada por Hibernação Miocárdica. Rev Bras Cardiol Invas. 2009;17(3):358-68.

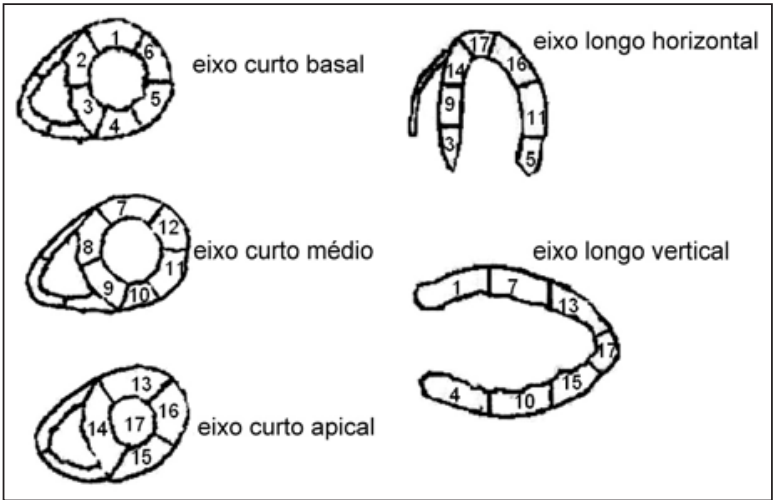

Figura 2 - Esquema de segmentação do ventrículo esquerdo para a cintilografia miocárdica SPECT: 1 = anterior basal; 2 = ânteroseptal basal; 3 = ínfero-septal basal; 4 = inferior basal; 5 = ínferolateral basal; 6 = ântero-lateral basal; $7=$ anterior médio; $8=$ ântero-septal médio; 9 = ínfero-septal médio; 10 = inferior médio; 11 = ínfero-lateral médio; 12 = ântero-lateral médio; 13 = ânteroapical; 14 = septo apical; 15 = ínfero-apical; 16 = látero-apical; 17 = apical.

Para ficar de acordo com o esquema de segmentação utilizado para a análise de mobilidade ventricular na ventriculografia de contraste, as pontuações de perfusão das seguintes lâminas foram assim consideradas: anterior basal $=$ segmento 1 ; ântero-lateral $=$ segmento 12 ; apical $=$ segmento 17 ; inferior $=$ segmento 10 ; inferior basal = segmento 4 (projeção da oblíqua anterior direita); ínfero-lateral basal = segmento 5; e ínfero-lateral médio = segmento 11 (projeção da oblíqua anterior esquerda). Para o segmento septal da ventriculografia por contraste na projeção da oblíqua anterior direita, considerou-se a combinação das pontuações dos segmentos 2 e 8 da cintilografia de perfusão.

\section{Análise estatística}

Os valores são apresentados como média \pm desvio padrão. Utilizou-se o teste de Wilcoxon para comparar os escores de mobilidade ventricular pareados nos batimentos anteriores e posteriores ao batimento ventricular ectópico. Para comparar a proporção de segmentos do ventrículo esquerdo apresentando padrões variáveis de perfusão miocárdica em pacientes com e sem potenciação pós-extrassistólica, utilizou-se o teste de qui-quadrado ou o teste de Fisher.

\section{RESULTADOS}

\section{Angiocoronariografia}

Todos os pacientes incluídos neste estudo tinham artérias coronárias normais na angiografia (20 pacientes) (Figura 3) ou lesões com redução $<50 \%$ do diâmetro luminal (2 pacientes).

\section{Ventriculografia de contraste}

A Tabela 1 apresenta as pontuações de mobilidade ventricular de todos os segmentos analisados para o batimento basal anterior e para o batimento após a extrassístole ventricular espontânea (representados, respectivamente, pelos algarismos posicionados antes e depois da barra) nas projeções oblíqua anterior direita e oblíqua anterior esquerda para todos os pacientes.

Em 14 dos 22 pacientes, no mínimo um segmento com discinergia apresentou melhora na pontuação de contração após a extrassístole ventricular, caracterizando a ocorrência de potenciação pós-extrassistólica. Três pacientes tiveram mais de um segmento com potenciação pós-extrassistólica. Dos 176 segmentos analisados, as pontuações de mobilidade ventricular melhoraram em 18 (10,2\%) segmentos, correspondendo a $14,6 \%$ dos 123 segmentos discinérgicos. O escore de mobilidade segmentar médio caiu em $8,7 \%$ (de $1,49 \pm 0,73$ para $1,36 \pm 0,76)$ para todo o grupo de pacientes (teste de Wilcoxon, $\mathrm{P}<0,05$ ) (Tabela 1). Observou-se potenciação pós-extrassistólica nos segmentos ventriculares apresentando vários graus de discinergia no período basal, de hipocinesia leve (5 segmentos), de hipocinesia moderada (5 segmentos) e de acinesia (8 segmentos), mas não nos segmentos que apresentaram discinesia no período basal. A região apical estava envolvida em 5 das 18 ocorrências, a região inferior basal estava envolvida em 4, seguida das regiões ântero-basal e ântero-lateral (3 ocorrências cada), e as regiões inferior, ínfero-lateral basal e ínfero-lateral média estavam envolvidas em uma ocorrência de potenciação pós-extrassistólica cada. O segmento septal do ventrículo esquerdo foi o único que não teve potenciação pós-extrassistólica.

A Figura 4 ilustra a potenciação pós-extrassistólica em segmento do ventrículo esquerdo em um paciente, na visão oblíqua anterior direita, e a Figura 5 apresenta a ausência de potenciação pós-extrassistólica em todos os segmentos observados na visão oblíqua anterior esquerda, no mesmo paciente.

\section{Cintilografia miocárdica}

No grupo de 14 pacientes que fizeram exercício por uma média de 10,2 $\pm 2,1$ METS, a frequência cardíaca aumentou de 73,6 \pm 13 no período basal para $143 \pm 16,1$ batimentos/minuto ao exercício máximo; a pressão arterial mudou de 128,1 $\pm 21,1 \mathrm{mmHg} /$ $80,6 \pm 11,2 \mathrm{mmHg}$ para 150,6 \pm 20,1 $\mathrm{mmHg} / 84,3 \pm$ $12,1 \mathrm{mmHg}$. No grupo que recebeu infusão de dipiridamol, a frequência cardíaca variou de 64,8 \pm 5,9 batimentos/minuto no período basal para 73,6 $\pm 8,4$ batimentos/minuto no final da infusão; nesse grupo, a alteração correspondente na pressão arterial foi de $134 \pm 16,7 \mathrm{mmHg} / 86 \pm 5,5 \mathrm{mmHg}$ no período basal para $114 \pm 15,2 \mathrm{mmHg} / 70 \pm 7,1 \mathrm{mmHg}$ no final da infusão. Os pacientes que receberam dobutamina como estressor apresentaram variação da frequência cardíaca de 66 batimentos/minuto para 150 batimentos/minuto e sua pressão arterial variou de 110/80 mmHg para 130/90 mmHg. Nenhum paciente em qualquer 


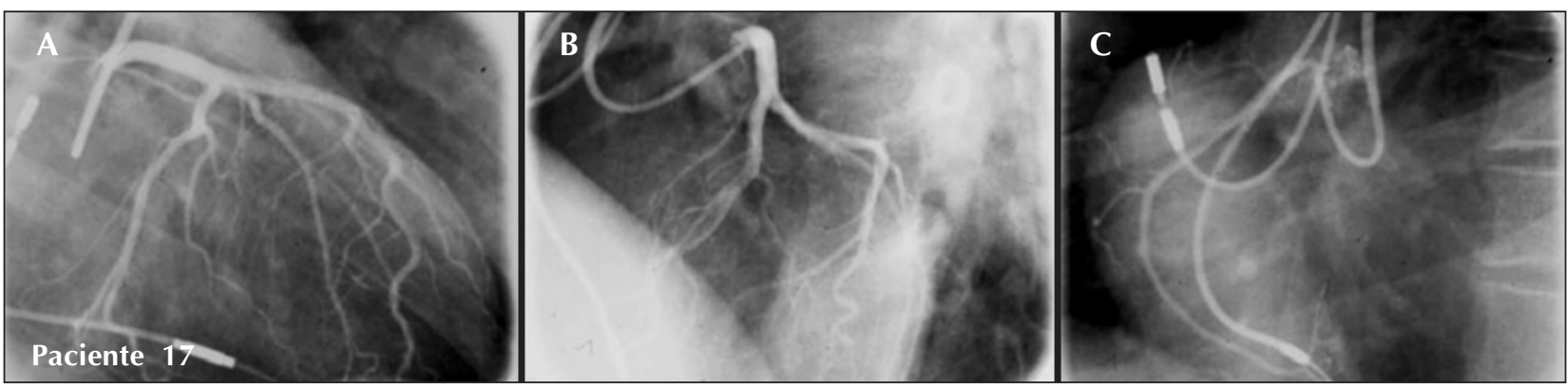

Figura 3 - Angiocoronariografia apresentando artérias epicárdicas normais no paciente 17. Em A, artéria coronária esquerda na visão oblíqua direita. Em B e C, artérias coronárias esquerda e direita, respectivamente, na visão oblíqua esquerda.

TABELA 1

Escores de mobilidade segmentar para cada segmento do ventrículo esquerdo durante a ventriculografia de contraste, nos batimentos imediatamente antes e após o batimento ventricular ectópico (representados pelos algarismos posicionados, respectivamente, antes e depois das barras)

\begin{tabular}{|c|c|c|c|c|c|c|c|c|c|}
\hline Paciente & $\mathrm{AB}$ & AL & Ap & In & IB & Sp & ILB & ILM & Média da pontuação de contração \\
\hline $1 *$ & $1 / 1$ & $1 / 1$ & $3 / 3$ & $1 / 1$ & $2 / 2$ & $2 / 2$ & $1 / 1$ & $3 / 3$ & $1,75 / 1,75$ \\
\hline $2^{*}$ & $0 / 0$ & $0 / 0$ & $4 / 4$ & $0 / 0$ & $0 / 0$ & $0 / 0$ & $0 / 0$ & $0 / 0$ & $0,50 / 0,50$ \\
\hline $3 *$ & $0 / 0$ & $0 / 0$ & $4 / 4$ & $1 / 1$ & $2 / 2$ & $0 / 0$ & $3 / 3$ & $1 / 1$ & $1,38 / 1,38$ \\
\hline $4^{*}$ & $2 / 1$ & $2 / 2$ & $3 / 3$ & $2 / 2$ & $3 / 3$ & $1 / 1$ & $1 / 1$ & $3 / 3$ & $2,13 / 2,00$ \\
\hline $5^{*}$ & $1 / 0$ & $1 / 0$ & $3 / 2$ & $0 / 0$ & $1 / 1$ & $0 / 0$ & $0 / 0$ & $1 / 1$ & $0,88 / 0,50$ \\
\hline $6^{*}$ & $0 / 0$ & $2 / 1$ & $3 / 3$ & $2 / 2$ & $3 / 2$ & $2 / 2$ & $0 / 0$ & $3 / 3$ & $1,88 / 1,63$ \\
\hline $7^{*}$ & $0 / 0$ & $1 / 1$ & $4 / 4$ & $1 / 1$ & $2 / 1$ & $1 / 1$ & $2 / 2$ & $2 / 2$ & $1,63 / 1,50$ \\
\hline $8^{*}$ & $1 / 1$ & $1 / 1$ & $3 / 2$ & $1 / 1$ & $2 / 2$ & $1 / 1$ & $3 / 3$ & $3 / 3$ & $1,88 / 1,75$ \\
\hline 9* & $0 / 0$ & $0 / 0$ & $3 / 2$ & $3 / 1$ & $1 / 1$ & $0 / 0$ & $4 / 4$ & $0 / 0$ & $1,38 / 1,00$ \\
\hline $10^{*}$ & $1 / 1$ & $2 / 2$ & $4 / 4$ & $2 / 2$ & $2 / 2$ & $1 / 1$ & $2 / 2$ & $3 / 3$ & $2,13 / 2,13$ \\
\hline $11 *$ & $1 / 1$ & $1 / 1$ & $3 / 3$ & $1 / 1$ & $4 / 4$ & $1 / 1$ & $3 / 1$ & $3 / 2$ & $2,13 / 1,75$ \\
\hline $12^{*}$ & $0 / 0$ & $0 / 0$ & $4 / 4$ & $0 / 0$ & $1 / 0$ & $0 / 0$ & $0 / 0$ & $1 / 1$ & $0,75 / 0,63$ \\
\hline $13^{*}$ & $0 / 0$ & $0 / 0$ & $4 / 4$ & $0 / 0$ & $1 / 1$ & $0 / 0$ & $0 / 0$ & $1 / 1$ & $0,75 / 0,75$ \\
\hline $14^{*}$ & $0 / 0$ & $0 / 0$ & $1 / 0$ & $0 / 0$ & $0 / 0$ & $0 / 0$ & $0 / 0$ & $0 / 0$ & $0,13 / 0,00$ \\
\hline $15^{*}$ & $1 / 1$ & $2 / 2$ & $3 / 3$ & $2 / 2$ & $3 / 3$ & $1 / 1$ & $2 / 2$ & $3 / 3$ & $2,13 / 2,13$ \\
\hline 16 & $2 / 2$ & $2 / 2$ & $4 / 4$ & $2 / 2$ & $3 / 3$ & $2 / 2$ & $3 / 3$ & $3 / 3$ & $2,63 / 2,63$ \\
\hline 17 & $0 / 0$ & $3 / 1$ & $3 / 3$ & $0 / 0$ & $1 / 1$ & $0 / 0$ & $0 / 0$ & $1 / 1$ & $1,00 / 0,75$ \\
\hline $18^{*}$ & $0 / 0$ & $0 / 0$ & $2 / 1$ & $0 / 0$ & $0 / 0$ & $0 / 0$ & $1 / 1$ & 0/0 & $0,38 / 0,25$ \\
\hline 19 & $1 / 1$ & $2 / 2$ & $4 / 4$ & $2 / 2$ & $3 / 3$ & $1 / 1$ & $3 / 3$ & $3 / 3$ & $2,38 / 2,38$ \\
\hline 20 & $0 / 0$ & $1 / 1$ & $3 / 3$ & $1 / 1$ & $2 / 2$ & $1 / 1$ & $3 / 3$ & $3 / 2$ & $1,75 / 1,63$ \\
\hline 21 & $0 / 0$ & $0 / 0$ & $4 / 4$ & $0 / 0$ & $1 / 0$ & $0 / 0$ & $0 / 0$ & $1 / 1$ & $0,75 / 0,63$ \\
\hline 22 & $2 / 1$ & $2 / 2$ & $4 / 4$ & $2 / 2$ & $2 / 2$ & $1 / 1$ & $3 / 3$ & $3 / 3$ & $\begin{array}{c}2,38 / 2,25 \\
1,49 \pm 0,73 / 1,36 \pm 0,76 \\
P<0,05^{* *}\end{array}$ \\
\hline
\end{tabular}

* Pacientes submetidos a estresse por esforço físico máximo.

** Média e desvio padrão da pontuação de contração de cada batimento e comparação pelo teste de Wilcoxon para o grupo inteiro. Destaques em negrito mostram os segmentos em que ocorreu potenciação pós-extrassistólica.

Segmentação do ventrículo esquerdo, de acordo com a nomenclatura utilizada na Figura 1: $\mathrm{AB}=$ anterior basal; $\mathrm{AL}=$ ântero-lateral; $\mathrm{Ap}=$ apical; IB = inferior basal; ILB = ínfero-lateral basal; ILM = ínfero-lateral médio; In = inferior; Sp = septal.

um dos grupos teve sintomas de angina. Em um paciente recebendo dipiridamol foi necessário administrar aminofilina após a injeção de tálio para reverter as marcantes alterações hemodinâmicas que ocorreram: queda da pressão arterial de 140/90 mmHg para 90/
$60 \mathrm{mmHg}$, acompanhada de taquicardia (55 batimentos/ minuto para 88 batimentos/minuto).

A Tabela 2 apresenta a pontuação durante as fases de estresse, redistribuição e reinjeção da cinti- 
Miziara A, et al. Discinergia Ventricular Esquerda Reversível Identificada por Potenciação Pós-Extrassistólica em Miocardiopatia Chagásica Crônica Não é Causada por Hibernação Miocárdica. Rev Bras Cardiol Invas. 2009;17(3):358-68.

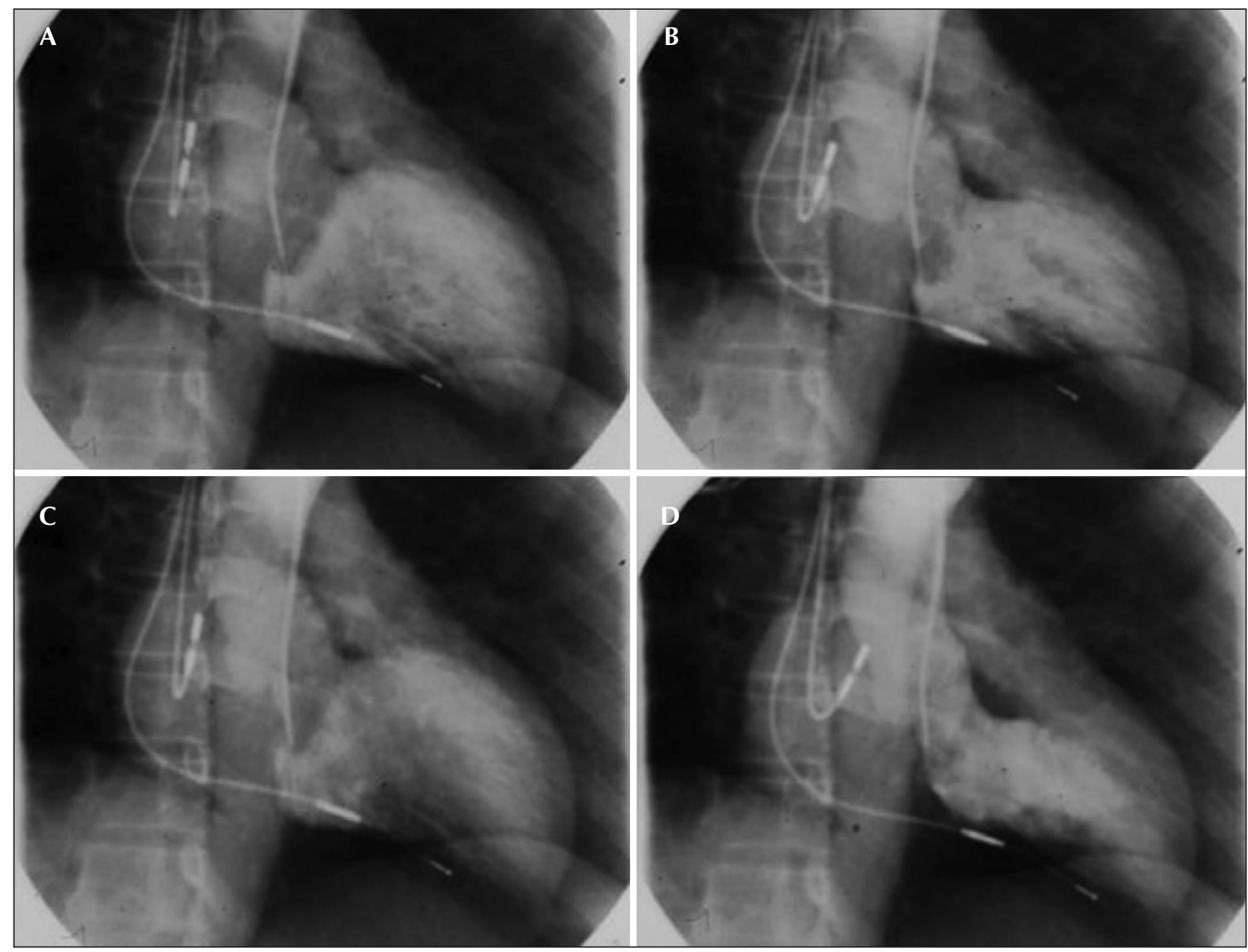

Figura 4 - Exemplo de potenciação pós-extrassistólica no segmento IV ântero-lateral (paciente 17). Em A e B, quadros do fim da diástole e do fim da sístole, respectivamente, do batimento anterior ao batimento ventricular ectópico. Em C e D, quadros do fim da diástole e do fim da sístole, respectivamente, do batimento imediatamente posterior ao batimento ventricular ectópico.

lografia miocárdica para os 18 segmentos que mostram potenciação pós-extrassistólica. A perfusão miocárdica foi totalmente normal durante estresse, redistribuição e reinjeção de tálio-201 na cintilografia miocárdica em 10 desses segmentos. Houve hipoperfusão fixa moderada em 4 segmentos e efeitos paradoxais (corrigidos por reinjeção) em outros 4 segmentos. Não detectamos hipoperfusão fixa grave nem isquemia reversível nesses segmentos com potenciação pósextrassistólica. A Figura 6 mostra o SPECT das fases de redistribuição e reinjeção da cintilografia miocárdica com tálio para o mesmo paciente das Figuras 3 a 5 .

\section{Comparação de grupos de pacientes com e sem potenciação pós-extrassistólica}

No grupo de 14 pacientes com pelo menos um segmento com potenciação pós-extrassistólica, 74 segmentos tinham discinergia (18 com e 56 sem potenciação pós-extrassistólica) e 38 (33,9\%) segmentos não tinham discinergia no período basal. No gru- po de 8 pacientes cujos segmentos não apresentavam potenciação pós-extrassistólica, 49 segmentos tinham discinergia e apenas $15(23,4 \%)$ não tinham discinergia.

A Tabela 3 mostra que o grupo com potenciação pós-extrassistólica teve maior proporção de segmentos com perfusão normal, com defeitos fixos leves ou com redistribuição reversa, em comparação com o grupo de pacientes que não tiveram potenciação pósextrassistólica, que apresentaram maior proporção de segmentos com defeitos fixos graves.

Considerando apenas segmentos sem discinergia no batimento basal em ambos os grupos, a Tabela 4 demonstra que eles tiveram proporções comparáveis de segmentos com perfusão normal. Os pacientes que apresentaram potenciação pós-extrassistólica tiveram maior número de segmentos com defeitos fixos leves e menor proporção de segmentos com defeitos reversíveis. A Tabela 4 também demonstra que, considerando apenas segmentos com discinergia em ambos 
Miziara A, et al. Discinergia Ventricular Esquerda Reversível Identificada por Potenciação Pós-Extrassistólica em Miocardiopatia Chagásica Crônica Não é Causada por Hibernação Miocárdica. Rev Bras Cardiol Invas. 2009;17(3):358-68.

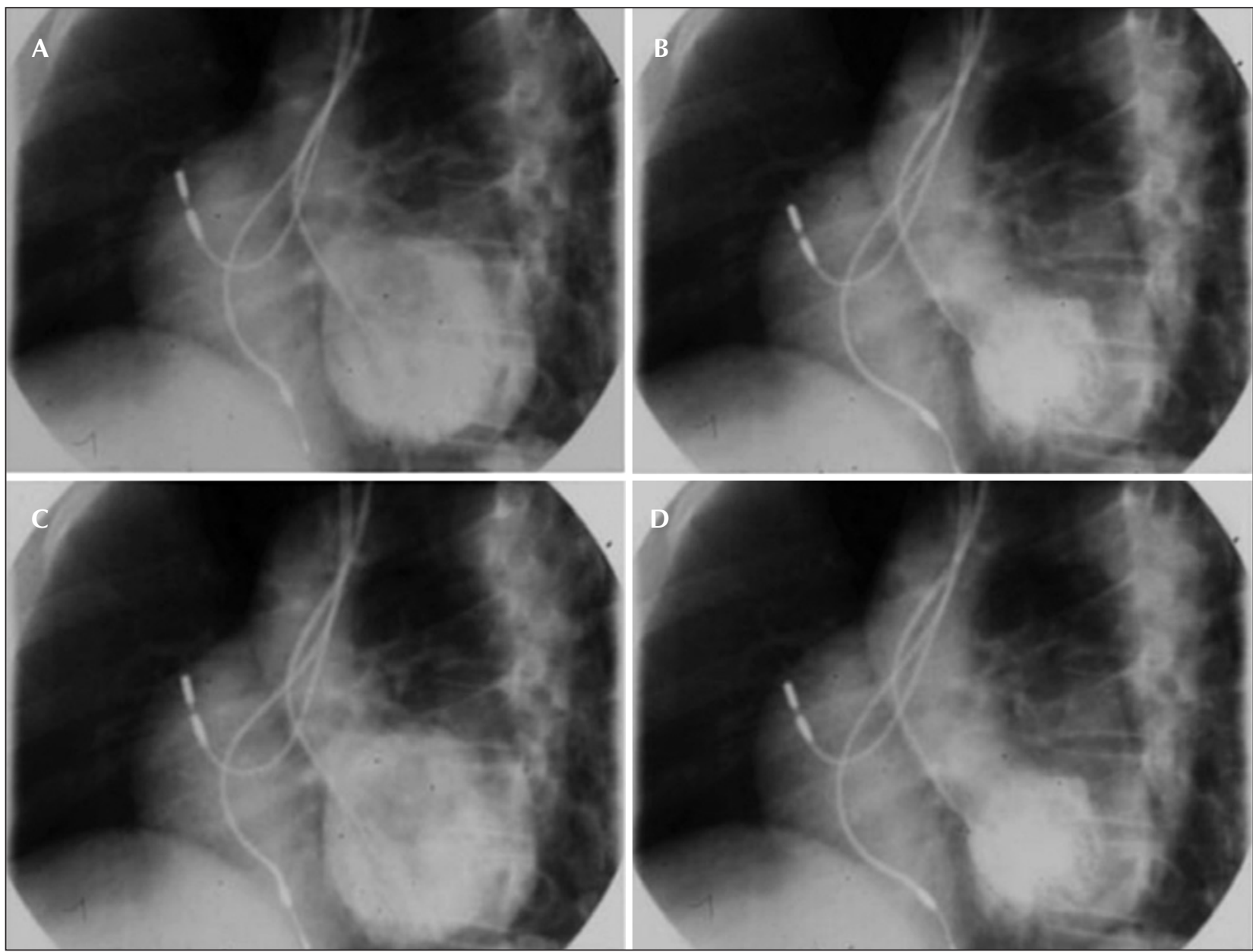

Figura 5 - Não foi observada potenciação pós-extrassistólica de qualquer um dos segmentos visualizados na visão oblíqua anterior esquerda do mesmo paciente (paciente 17). A apresentação dos painéis A, B, C e D é a mesma utilizada na Figura 4.

os grupos, o grupo que apresentava potenciação pósextrassistólica teve maior proporção de segmentos com perfusão normal, defeitos fixos leves ou redistribuição reversa e menor proporção de segmentos com defeitos fixos graves.

A Tabela 5 apresenta comparação do padrão de perfusão para todos os segmentos com discinergia, conforme a presença ou a ausência de potenciação pósextrassistólica. Nos segmentos com discinergia sem potenciação pós-extrassistólica, mas não naqueles com potenciação pós-extrassistólica, foram registrados padrões de defeitos fixos graves e de defeitos reversíveis, enquanto a proporção de padrões de perfusão normal, defeitos fixos leves e redistribuição reversa foi semelhante.

\section{DISCUSSÃO}

Já se demonstrou que o fenômeno fisiológico da potenciação pós-extrassistólica depende, essencialmente, do aumento da contratilidade intrínseca após a extrassístole e, em menor extensão, da pré-carga au- mentada que normalmente ocorre durante o período diastólico ${ }^{10-12}$. A demonstração da potenciação pósextrassistólica em pacientes com doença cardíaca isquêmica foi o primeiro método usado clinicamente para desmascarar o miocárdio viável em segmentos com discinergia ventricular previamente considerados necróticos ou fibróticos ${ }^{13}$. Realizada primeiramente durante a ventriculografia por contraste ${ }^{14}$, a potenciação pós-extrassistólica baseia-se no conceito de recrutamento de reserva contrátil em áreas discinérgicas, porém viáveis. Posteriormente, foi usada durante a ecocardiografia para a avaliação da função ventricular esquerda regional e global ${ }^{15,16}$.

Recentemente demonstrou-se que a potenciação pós-extrassistólica ocorre em um número substancial de segmentos com discinergia do ventrículo esquerdo em pacientes com miocardiopatia chagásica crônica. Este estudo amplia esses achados, demonstrando que a potenciação pós-extrassistólica detectada em segmentos do ventrículo esquerdo com vários graus de discinergia (exceto a discinesia) ocorre em áreas mio- 
Miziara A, et al. Discinergia Ventricular Esquerda Reversível Identificada por Potenciação Pós-Extrassistólica em Miocardiopatia Chagásica Crônica Não é Causada por Hibernação Miocárdica. Rev Bras Cardiol Invas. 2009;17(3):358-68.

TABELA 2

Padrão de perfusão da cintilografia miocárdica em segmentos do ventrículo esquerdo apresentando potenciação pós-extrassistólica durante ventriculografia de contraste

\begin{tabular}{lclll}
\hline Paciente & Segmento do ventrículo esquerdo & E & Rd & RI \\
\hline 4 & Anterior basal & 0 & 0 & 0 \\
5 & Anterior basal & 0 & 0 & 0 \\
& Antero-lateral & 0 & 0 & 0 \\
Apical & 0 & 0 & 0 \\
6 & Antero-lateral & 0 & 0 & 0 \\
& Inferior basal & 0 & 2 & 1 \\
7 & Inferior basal & 0 & 0 & 0 \\
8 & Apical & 1 & 1 & 1 \\
9 & Apical & 2 & 2 & 0 \\
\multicolumn{1}{c}{ Inferior } & 0 & 0 & 1 \\
11 & Ínfero-lateral basal & 2 & 3 & 0 \\
12 & Inferior basal & 0 & 0 & 0 \\
17 & Apical & 0 & 1 & 1 \\
18 & Antero-lateral & 0 & 0 & 1 \\
20 & Apical & 1 & 1 & 1 \\
21 & Ínfero-lateral médio & 1 & 1 & 0 \\
22 & Inferior basal & 0 & 2 & 0 \\
\hline
\end{tabular}

$\mathrm{E}=$ estresse; $\mathrm{Rd}=$ redistribuição; $\mathrm{RI}=$ reinjeção de tálio-201 em repouso.

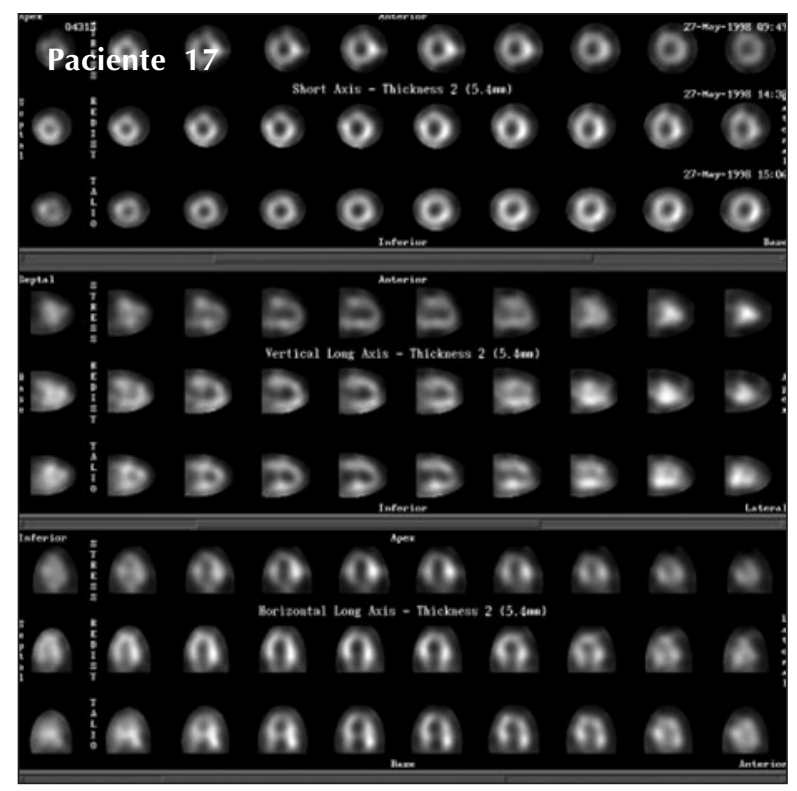

Figura 6 - Resultados de cintilografia miocárdica com tálio-201 SPECT para o mesmo paciente das Figuras 3 a 5 (paciente 17), cuja ventriculografia de contraste é apresentada nas Figuras 4 e 5. A perfusão é totalmente normal durante as fases de estresse, redistribuição e reinjeção normal no segmento ântero-lateral (segmento 12), apresentando potenciação pós-extrassistólica. Observa-se um defeito de perfusão moderado no segmento anterior médio (segmento 7) durante estresse, parcialmente reversível após a redistribuição e totalmente reversível com a reinjeção de tálio. Observa-se um defeito fixo leve no segmento apical (segmento 17). cárdicas essencialmente viáveis, cuja perfusão é normal ou apenas moderadamente alterada não somente no período basal, mas também sob estresse máximo. Por outro lado, e ao contrário da hipótese formulada no início desta pesquisa, a potenciação pós-extrassistólica não poderia ser demonstrada em áreas ventriculares discinérgicas cuja perfusão estava prejudicada no período basal ou com isquemia induzida durante o estresse. Assim, os resultados desta pesquisa não corroboram a hipótese de que poderia haver áreas de discinergia ventricular em pacientes com miocardiopatia chagásica crônica em decorrência de uma forma de hibernação miocárdica causada por distúrbios microvasculares. É notável que, utilizando ecocardiograma de estresse induzido por dobutamina, outros pesquisadores descreveram reserva contrátil limitada em pacientes com miocardiopatia chagásica: alguns pacientes chagásicos apresentaram resposta bifásica que melhorou com o uso de baixas doses e deteriorou com a dose máxima, achados semelhantes àqueles observados em pacientes com doença coronária. Esses pesquisadores também sugeriram que a isquemia pode contribuir para a disfunção do ventrículo esquerdo na miocardiopatia chagásica ${ }^{17}$.

\section{CONCLUSÃO}

Em suma, os resultados deste estudo em pacientes chagásicos indicam que: a) a potenciação pós-extras- 
Miziara A, et al. Discinergia Ventricular Esquerda Reversível Identificada por Potenciação Pós-Extrassistólica em Miocardiopatia Chagásica Crônica Não é Causada por Hibernação Miocárdica. Rev Bras Cardiol Invas. 2009;17(3):358-68.

TABELA 3

Padrão de perfusão miocárdica em pacientes com e sem potenciação pós-extrassistólica

\begin{tabular}{lcc}
\hline & Pacientes com PEP & Pacientes sem PEP \\
\hline Perfusão normal & $66(58,9 \%)$ & $30(46,9 \%)$ \\
Defeitos fixos leves & $21(18,8 \%)$ & $8(12,5 \%)$ \\
Defeitos fixos graves & $6(5,3 \%)$ & $18(28,1 \%)$ \\
Defeitos reversíveis & $9(8 \%)$ & $7(10,9 \%)$ \\
Redistribuição reversa & $10(8,9 \%)$ & $1(1,6 \%)$ \\
\hline Os números referem-se a segmentos com cada padrão de perfusão. Os valores porcentuais referem-se ao número total de segmentos \\
analisados em cada grupo. O padrão de perfusão é significativamente diferente entre os dois grupos $\left(\chi^{2}=21,45 ; P<0,001\right)$. \\
PEP = potenciação pós-extrassistólica.
\end{tabular}

TABELA 4

Padrão de perfusão miocárdica em segmentos apresentando ou não discinergia basal em pacientes com e sem potenciação pós-extrassistólica

\begin{tabular}{|c|c|c|c|c|}
\hline & \multicolumn{2}{|c|}{ Segmentos sem discinergia* } & \multicolumn{2}{|c|}{ Segmentos com discinergia** } \\
\hline & Pacientes com PEP & Pacientes sem PEP & Pacientes com PEP & Pacientes sem PEP \\
\hline Perfusão normal & $28(73,7 \%)$ & $11(73,3 \%)$ & $38(51,4 \%)$ & $19(38,8 \%)$ \\
\hline Defeitos fixos leves & $6(15,8 \%)$ & $1(6,7 \%)$ & $15(20,3 \%)$ & $7(14,3 \%)$ \\
\hline Defeitos fixos graves & 0 & 0 & $6(8,1 \%)$ & $18(36,7 \%)$ \\
\hline Defeitos reversíveis & $3(7,9 \%)$ & $3(20,0 \%)$ & $6(8,1 \%)$ & $4(8,2 \%)$ \\
\hline Redistribuição reversa & $1(2,6 \%)$ & 0 & $9(12,2 \%)$ & $1(2,0 \%)$ \\
\hline \multicolumn{5}{|c|}{$\begin{array}{l}\text { * Entre os segmentos sem discinergia, não houve diferença significativa entre o padrão de perfusão dos pacientes com PEP e sem PEP } \\
\left(\chi^{2}=3,34 ; P=0,42\right) \text {. } \\
* * \text { Entre os segmentos com discinergia, houve diferença significativa entre o padrão de perfusão dos pacientes com PEP e sem PEP } \\
\left(\chi^{2}=17,69 ; P=0,001\right) \text {. } \\
\text { Os números referem-se a segmentos com cada padrão de perfusão. Os valores porcentuais referem-se ao número total de segmentos } \\
\text { analisados em cada grupo. } \\
\text { PEP = potenciação pós-extrassistólica. }\end{array}$} \\
\hline
\end{tabular}

TABELA 5

Padrão de perfusão miocárdica em todos os segmentos com discinergia em pacientes com e sem potenciação pós-extrassistólica

\begin{tabular}{|c|c|c|}
\hline & $\begin{array}{l}\text { Segmentos com discinergia } \\
\text { em pacientes com PEP }\end{array}$ & $\begin{array}{l}\text { Segmentos com discinergia } \\
\text { em pacientes sem PEP }\end{array}$ \\
\hline Perfusão normal & $10(55,6 \%)$ & $28(50,0 \%)$ \\
\hline Defeitos fixos leves & $4(22,2 \%)$ & $11(19,6 \%)$ \\
\hline Defeitos fixos graves & 0 & $6(10,7 \%)$ \\
\hline Defeitos reversíveis & 0 & $6(10,7 \%)$ \\
\hline \multicolumn{3}{|c|}{$\begin{array}{l}\text { Os números referem-se a segmentos com cada padrão de perfusão. Os valores porcentuais referem-se ao número total de segmentos } \\
\text { analisados com ou sem potenciação pós-extrassistólica. Não houve diferença significativa entre os dois grupos }\left(\chi^{2}=5,96 ; \mathrm{P}=0,20\right) \text {. } \\
\text { PEP = potenciação pós-extrassistólica. }\end{array}$} \\
\hline
\end{tabular}

sistólica ocorreu somente em áreas discinérgicas do ventrículo esquerdo compostas de miocárdio essencialmente viável e normoperfundido; b) embora pos- sam ser detectados tanto em modelos experimentais ${ }^{18,19}$ quanto em pacientes com miocardiopatia chagásica crônica ${ }^{20-23}$, os distúrbios de perfusão microvascular 
Miziara A, et al. Discinergia Ventricular Esquerda Reversível Identificada por Potenciação Pós-Extrassistólica em Miocardiopatia Chagásica Crônica Não é Causada por Hibernação Miocárdica. Rev Bras Cardiol Invas. 2009;17(3):358-68.

não têm relação causal com a alteração regional da mobilidade segmentar do ventrículo esquerdo, tão característica e comum nessa doença. Entretanto, os atuais resultados em pacientes chagásicos, cuja principal manifestação clínica tenha sido a ocorrência de arritmias ventriculares, confirmam estudos prévios em pacientes chagásicos com sintomas semelhantes aos da angina ${ }^{3,21-24}$. Em ambos os grupos, na ausência de doença coronária obstrutiva epicárdica significativa, foram observados defeitos de perfusão marcantes de todos os tipos (fixos, reversíveis e paradoxais) 2,3,21-23.

É plausível especular que as alterações inflamatórias diretamente causadas pela presença de parasita ${ }^{25}$ ou mediadas por mecanismos imunológicos ${ }^{26}$ poderiam ser responsáveis pelos distúrbios regionais da mobilidade segmentar que ocorrem em áreas ventriculares, cuja reserva contrátil pode ser desmascarada pela potenciação pós-extrassistólica. Segundo essa hipótese, durante os estágios iniciais do processo inflamatório, a discinergia poderia ser causada por músculo cardíaco disfuncional, porém essencialmente viável, cuja reserva contrátil pode ser induzida durante o estímulo da potenciação pós-extrassistólica. Mesmo depois de estágios mais avançados do processo inflamatório, a área discinérgica no período basal pode ser composta de tecido fibrótico mesclado com miocárdio normal: a reserva contrátil desse componente normal poderia, portanto, ser desmascarada por estímulo pósextrassistólico. Finalmente, não é aceitável descartar completamente a possível contribuição de um mecanismo suplementar para potenciação pós-extrassistólica detectada em miocardiopatas chagásicos: semelhante ao que é postulado para pacientes com doença arterial coronária, durante a contração mais potente de áreas ventriculares contíguas evocadas pela potenciação pós-extassistólica, fibras do miocárdio não-viável discinérgico são arrastadas em conjunto, o que pode ser falsamente interpretado como melhora da própria área $\operatorname{afetada}^{27,28}$

Finalmente, embora os presentes achados não corroborem a hipótese da hibernação miocárdica na miocardiopatia chagásica crônica, a noção de que os distúrbios microvasculares desempenham ao menos papel ancilar nessa patogênese ${ }^{29}$ foi recentemente ratificada pela demonstração de um decréscimo paralelo da função sistólica do ventrículo esquerdo e da conversão de defeitos miocárdicos previamente isquêmicos em alterações de perfusão irreversíveis (fibróticas) ${ }^{30}$.

\section{CONFLITO DE INTERESSES}

Os autores declararam inexistência de conflito de interesses relacionados a este artigo.

\section{REFERÊNCIAS BIBLIOGRÁFICAS}

1. Marin-Neto JA, Simões MV, Maciel BC. Specific diseases: cardiomyopathies and pericardial diseases. Other cardio- myopathies. In: Yusuf S, Cairns JA, Camm AJ, Fallen EL, Gersh BJ, editors. Evidence-based cardiology. $2^{\text {nd }}$ ed. London: BMJ Books, Brit Med Association; 2003. p. 718-73.

2. Sarabanda $A V$, Sosa $E$, Simões $M V$, Figueiredo GL, Pintyan AO, Marin-Neto JA. Ventricular tachycardia in Chagas' disease: a comparison of clinical, angiographic, electrophysiologic and myocardial perfusion disturbances between patients presenting with either sustained or nonsustained forms. Int J Cardiol. 2005;102(1):9-19.

3. Marin-Neto JA, Marzullo P, Sousa AC, Marcassa C, Macie $B C$, lazigi $N$, et al. Radionuclide angiographic evidence for early predominant right ventricular involvement in patients with Chagas' disease. Can J Cardiol. 1988;4(5):231-6.

4. Marin-Neto JA, Bromberg-Marin G, Pazin-Filho A, Simões MV, Maciel BC. Cardiac autonomic impairment and early myocardial damage involving the right ventricle are independent phenomena in Chagas' disease. Int J Cardiol. 1998;65(3):261-9.

5. Brandão JM, Miziara A, Figueiredo GL, Lima-Filho $M O$ Ayres-Neto EM, Marin-Neto JA. Post-extrasystolic potentiation in chronic Chagas' heart disease. A radiologic contrast ventriculography study. Arq Bras Cardiol. 2005;84(5):376-80.

6. Rahimtoola $\mathrm{SH}$. The hibernating myocardium. Am Heart J. 1989;117(1):211-21.

7. Udelson JE, Bonow RO, Dilsizian V. The historical and conceptual evolution of radionuclide assessment of myocardial viability. J Nucl Cardiol. 2004;11(3):318-34.

8. Principal Investigators of CASS and Their Associates. The National Heart, Lung and Blood Institute Coronary Artery Surgery Study. A multicenter comparison of the effects of randomized medical and surgical treatment of mildly symptomatic patients with coronary artery disease, and a registry of consecutive patients undergoing coronary angiography. Circulation. 1981;63(6 Pt 2):11-81.

9. Cerqueira MD, Weissman NJ, Dilsizian V, Jacobs AK, Kaul S, Laskey WK, et al; American Heart Association Writing Group on Myocardial Segmentation and Registration for Cardiac Imaging. Standardized myocardial segmentation and nomenclature for tomographic imaging of the heart: a statement for healthcare professionals from the Cardiac Imaging Committee of the Council on Clinical Cardiology of the American Heart Association. J Nucl Cardiol. 2002;9(2):240-5.

10. der Werf T, van Poelgeest R, Herbschleb HH, Meijler FL. Postextrasystolic potentiation in man. Eur J Cardiol. 1976;4 Suppl:131-41.

11. Cooper MW. Postextrasystolic potentiation. Do we really know what it means and how to use it? Circulation. 1993; 88(6):2962-71

12. Vassallo DV, Lima EQ, Campagnaro P, Faria AN, Mill JG. Mechanisms underlying the genesis of post-extrasystolic potentiation in rat cardiac muscle. Braz J Med Biol Res. 1995;28(3):377-83.

13. Hamby RI, Aintablian A, Wisoff G, Hartstein ML. Response of the left ventricle in coronary artery disease to postextrasystolic potentiation. Circulation. 1975;51(3):428-35.

14. Banka VS, Bodenheimer MM, Shah R, Helfant RH. Intervention ventriculography. Comparative value of nitroglycerin, postextrasystolic potentiation and nitroglycerin plus post-extrasystolic potentiation. Circulation. 1976;53(4):632-7.

15. Cohn PF, Angoff GH, Zoll PM, Sloss LJ, Markis JE, Graboys $T B$, et al. A new, noninvasive technique for inducing postextrasystolic potentiation during echocardiography. Circulation. 1977;56(4 Pt 1):598-605

16. Scognamiglio R, Negut C, Palisi M. Spontaneous recovery of myocardial asynergic segments following acute myocardial infarction. The role of post-extrasystolic potentiation echo- 
Miziara A, et al. Discinergia Ventricular Esquerda Reversível Identificada por Potenciação Pós-Extrassistólica em Miocardiopatia Chagásica Crônica Não é Causada por Hibernação Miocárdica. Rev Bras Cardiol Invas. 2009;17(3):358-68.

cardiography in the predischarge evaluation. Eur J Echocardiogr. 2003;4(2):135-40.

17. Acquatella H, Pérez JE, Condado JA, Sánchez I. Limited myocardial contractile reserve and chronotropic incompetence in patients with chronic Chagas' disease: assessment by dobutamine stress echocardiography. J Am Coll Cardiol. 1999; 33(2):522-9

18. Figueiredo F, Marin-Neto JA, Rossi MA. The evolution of experimental Trypanosoma cruzi cardiomyopathy in rabbits: further parasitological, morphological and functional studies. Int J Cardiol. 1986;10(3):277-90.

19. Morris SA, Tanowitz HB, Wittner M, Bilezikian JP. Pathophysiological insights into the cardiomyophathy of Chagas' disease. Circulation. 1990;82(6):1900-9.

20. Rossi MA. Microvascular changes as a cause of chronic cardiomyopathy in Chagas' disease. Am Heart J. 1990; 120(1):233-6

21. Marin-Neto JA, Marzullo P, Marcassa C, Gallo Júnior L, Maciel BC, Bellina CR, et al. Myocardial perfusion abnormalities in chronic Chagas' disease as detected by thallium-201 scintigraphy. Am J Cardiol. 1992;69(8):780-4.

22. Hagar JM, Rahimtoola SH. Chagas' heart disease in the United States. N Engl J Med. 1991;325(11):763-8.

23. Simões MV, Pintya AO, Bromberg-Marin G, Sarabanda AV, Antloga CM, Pazin-Filho A, et al. Relation of regional sympathetic denervation and myocardial perfusion disturbance to wall motion impairment in Chagas' cardiomyopathy. Am J Cardiol. 2000;86(9):975-81.
24. Kuschnir E, Kustich F, Epelman MN, Pódio RB. Estúdios de flujo miocárdico en pacientes con insuficiencia coronaria y en pacientes con cardiopatia chagasica crónica: Respuesta a la administración de dinitrato de isosorbide y dipiridamol. Pren Med Arg. 1983;60:637-50.

25. Higuchi ML, Morais CF, Pereira Barreto AC, Lopes EA, Stolf $\mathrm{N}$, Bellotti $\mathrm{G}$, et al. The role of active myocarditis in the development of heart failure in chronic Chagas' disease: a study based on endomyocardial biopsies. Clin Cardiol. 1987;10(11):665-70.

26. Cunha-Neto E, Coelho V, Guilherme L, Fiorelli A, Stolf N, Kalil J. Autoimmunity in Chagas' disease. Identification of cardiac myosin-B13 Trypanosoma cruzi protein crossreactive T cell clones in heart lesions of a chronic Chagas' cardiomyopathy patient. J Clin Invest. 1996;98(8):1709-12.

27. Bach DS, Muller DW, Gros BJ, Armstrong WF. False positive dobutamine stress echocardiograms: characterization of clinical echocardiographic and angiographic findings. J Am Coll Cardiol. 1994;24(4):928-33.

28. Chia KK, Picard MH, Skopicki HA, Hung J. Viability of hypokinetic segments: influence of tethering from adjacent segments. Echocardiography. 2002;19(6):475-81.

29. Marin-Neto JA, Cunha-Neto E, Maciel BC, Simões MV. Pathogenesis of chronic Chagas heart disease. Circulation. 2007;115(9):1109-23.

30. Hiss FC, Lascala TF, Maciel BC, Marin-Neto JA, Simões MV. Changes in myocardial perfusion correlate with deterioration of left ventricular systolic function in chronic Chagas' cardiomyopathy. JACC Cardiovasc Imaging. 2009;2(2):164-72. 